\title{
Visual Reproduction II Subtest (WMS-IV)
}

National Cancer Institute

\section{Source}

National Cancer Institute. Visual Reproduction II Subtest (WMS-IV). NCI Thesaurus. Code C120349.

A subtest of the Wechsler Memory Scale, 4th Edition that assesses long-term visualspatial memory for nonverbal visual stimuli. A series of five designs is shown, one at a time, for 10 seconds each. After a delay, the subject is asked to draw the designs shown in any order. Then, the subject is asked to choose which of six designs on a page matches the original design shown. 